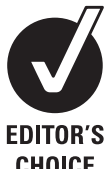

CHOICE

\title{
Sessile serrated adenomas: prevalence of dysplasia and carcinoma in 2139 patients
}

\author{
Richard H Lash, Robert M Genta, Christopher M Schuler
}

Caris Research Institute, Irving, Texas, USA

\section{Correspondence to} Richard H Lash, MD, Caris Research Institute, 6655 MacArthur Blvd, Irving, TX 75039, USA; rlash@carisdx.com

Accepted 11 March 2010 Published Online First 14 June 2010

\begin{abstract}
Background and aims Sessile serrated adenomas (SSAs) are recognised as precursors to microsatellite unstable adenocarcinomas. This study attempts to estimate the progression rate of SSAs based upon the epidemiology of a large cohort as well as identify relationships to other colorectal polyps.

Methods Pathological reports generated at Caris Diagnostics from 290810 colonoscopic specimens on 179111 patients were analysed using computerised algorithms.
\end{abstract}

Results SSAs with or without dysplasia/carcinoma (SSA $+/-$ ) were identified in 2416 specimens from 2139 patients (54\% women). The distribution of SSA+/was: right-sided (81.2\%); left-sided (11.2\%); both rightand left-sided (3.2\%); not specified (4.3\%). There were 1816 (85\%) patients without dysplasia (SSA-), 257 (12\%) with low-grade dysplasia (SSA-LD), 45 (2\%) with high-grade dysplasia (SSA-HD) and $21(1 \%)$ with adenocarcinoma (SSA-CA). The difference in median age between almost all groups was significant (SSA $-=61$ years versus $S S A-L D=66$ years $(p<0.001)$ vs $S S A-H D=72$ years $(p=0.002)$ vs $S S A-C A=76$ years $(p=0.07, N S))$. Women comprised $53 \%$ of the SSAgroup (968/1816), 57\% of the SSA-LD group (147/257), $69 \%$ of the SSA-HD group (31/45) and $76 \%$ of the SSACA group (16/21), being more likely to have high-grade dysplasia (OR 1.94, 95\% Cl 1.03 to 3.67) and adenocarcinoma (OR 2.80, 95\% Cl 1.02 to 7.68 ).

Conclusions $1.7 \%$ of patients with mucosal polyps had SSAs (with and without dysplasia), more commonly in women and primarily in the right colon. Dysplasia or carcinoma was identified in 15\% of patients and significantly disproportionately among women. Based on significant age differences between groups, there appears to be a stepwise progression of dysplasia and carcinoma in SSAs over 10 to 15 years, a period two to three times longer than that for conventional adenomas.

\section{INTRODUCTION}

Sessile serrated adenomas (SSAs) are increasingly recognised polyps that have been shown to have unique molecular alterations and histological features. ${ }^{1}$ These lesions have also been referred to as sessile serrated polyps due to the absence of adenoma-like dysplasia. In fact, at the December 2009 WHO meeting in Lyon, the combined term SSA/P was suggested; nevertheless, for the remainder of this manuscript, the term 'sessile serrated adenoma' (SSA) will be used for simplicity. A subset of these lesions develop histological dysplasia and carcinoma, ${ }^{2}{ }^{3}$ and there has been speculation that the development of adenocarcinoma in these polyps may be rapid. ${ }^{3-5}$ Some authors suggest that SSAs may explain the phenomenon of adenocarcinomas arising between scheduled surveillance colonoscopies ('interval' cancers). ${ }^{6}$ Unfortunately, disagreements in terminology, inconsistent application of histological criteria and insufficient large-scale studies have made it difficult to answer important questions about the behaviour, proper management and relationship of these lesions to other polyps of the colorectum. To evaluate the demographic characteristics of patients with SSAs, assess the prevalence of dysplasia and carcinoma associated with these polyps and discern associations of SSAs with other polyps of the colorectum, we retrospectively analysed diagnostic reports characterising a large sample of colorectal polyps evaluated in a consensusbased environment designed to yield consistent application of diagnostic criteria.

\section{METHODS}

\section{Study setting}

This study was approved by the Caris Diagnostics Institutional Review Board. Colorectal biopsies from Caris Diagnostics, a subspecialty gastrointestinal pathology laboratory receiving specimens from gastroenterologists operating in outpatient endoscopy centres in 36 states, the District of Columbia and Puerto Rico, were routinely fixed in $10 \%$ buffered formalin and embedded in paraffin prior to sectioning. Slides were prepared with $4 \mu \mathrm{m}$ thick sections and stained with H\&E. Interpretations were performed by an experienced group of gastrointestinal pathologists who share a common approach to biopsy evaluation and daily conferencing to review criteria and interpretation of problematic cases.

\section{Histopathological criteria}

Among serrated polyps, SSAs were recognised by their architectural and (secondarily) cytological features. These lesions were, by definition, sessile, with lateral serration of the glandular epithelium and branching and horizontal growth of crypt bases (figure 1). Other histological features supporting the diagnosis of SSA were maintenance of basal goblet cells ${ }^{1}$ and the presence of mitotic figures in the middle and upper third of the crypts. ${ }^{2}$ When there was low-grade dysplasia (figure 2), characterised by nuclear hyperchromasia and elongation, pseudostratification, apoptosis, increased mitotic activity, and loss of mucin as is seen in conventional adenomas, the polyp was classified as SSA with low-grade dysplasia (SSA-LD). When there was high-grade dysplasia (figures 3,4 ), characterised by greater nuclear enlargement, with nucleoli, loss of polarity and accompanying architectural complexity (eg, cribriforming), the polyp 


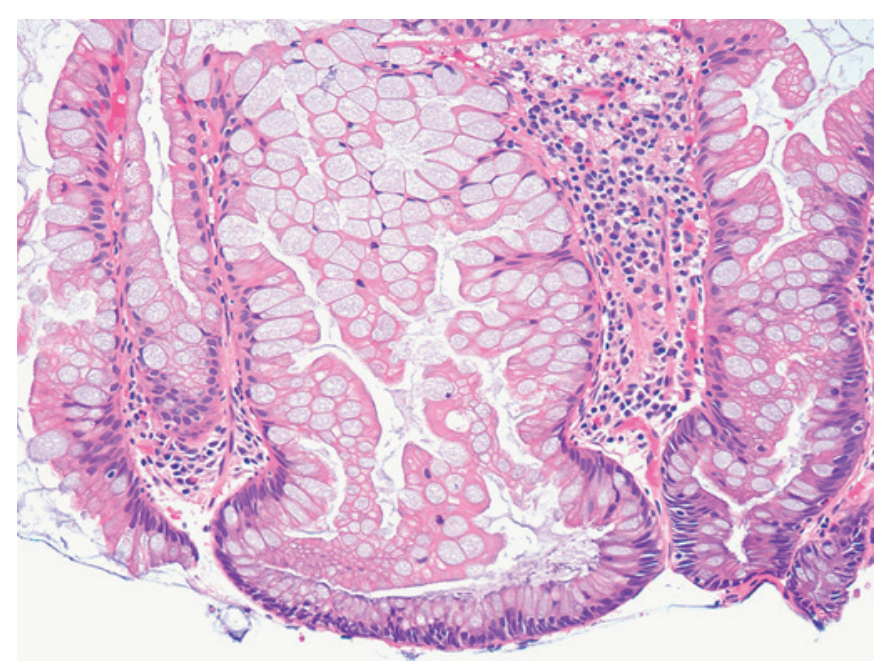

Figure 1 Sessile serrated adenoma (SSA): lateral serration of the glandular epithelium with branching and horizontal growth of crypt bases. Note the maintenance of basal goblet cells.

was classified as SSA with high-grade dysplasia (SSA-HD). Rarely, the dysplasia resembled that of a traditional serrated adenoma, including more uniformly enlarged nuclei, eosinophilic cytoplasm and the architectural presence of so-called ectopic crypt formation. ${ }^{7} 8$ Invasive adenocarcinomas arising within identifiable SSAs (typically also containing low- and high-grade dysplasia) were designated SSA-CA.

Hyperplastic polyps (HP) were defined as polyps with predominantly superficial serrations, narrow basilar crypt regions, proliferative zones limited to the lower portions of the crypts and no cytological dysplasia. For the purpose of this analysis, polyps with some characteristics considered suggestive but not diagnostic of SSA (typically rare dilated or distorted crypts in a somewhat sessile polyp) were amalgamated with the hyperplasic polyps. The term 'traditional serrated adenoma' (TSA) was used to describe serrated polyps with columnar configuration of the glandular epithelium, eosinophilic cytoplasm and uniform, non-stratified glandular dysplasia.

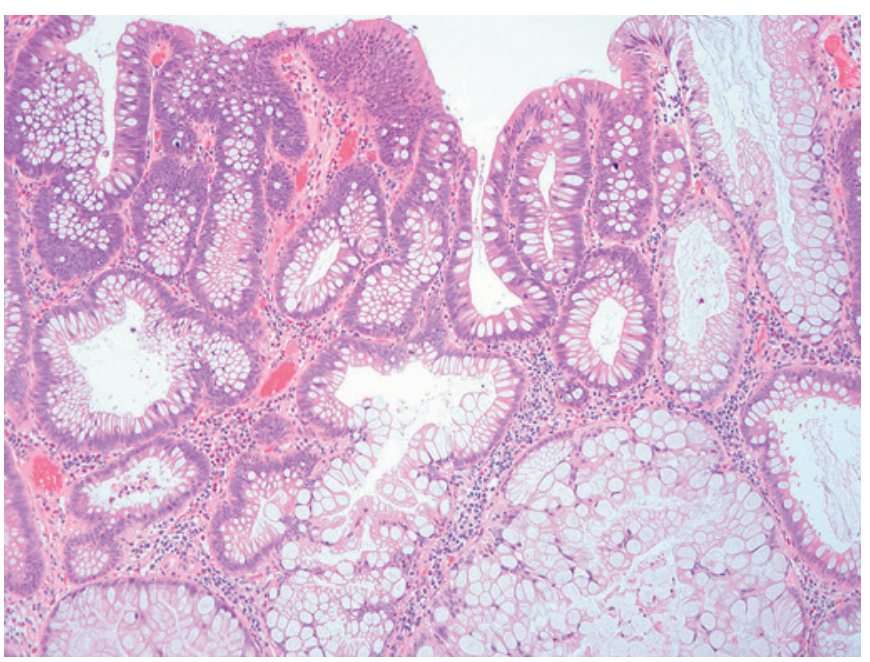

Figure 2 Sessile serrated adenoma with low-grade dysplasia. The columnar cells lining the surface and part of the glands have hyperchromatic, pseudostratified, pencillate nuclei; they also show apoptosis, increased mitotic activity and loss of mucin, as seen in conventional adenomas.

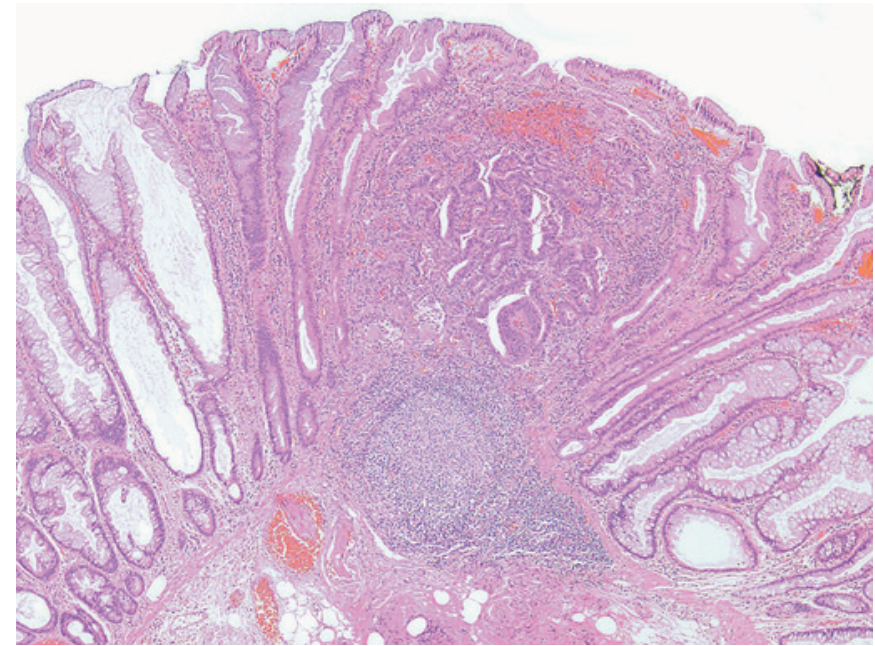

Figure 3 Sessile serrated adenoma with high-grade dysplasia. This low-power photomicrograph shows a serrated adenoma with a dysplastic focus (immediately above a lymphoid follicle) characterised by greater nuclear enlargement, loss of polarity and considerable architectural complexity.

Adenomatous polyps were recognised by conventional-type surface epithelial proliferation and dysplasia in which the columnar cells lining the polyps had hyperchromatic, pseudostratified, pencillate nuclei. These polyps were subclassified based upon architectural features (villous and tubulovillous) and the presence or absence of high-grade dysplasia. The resulting groups, based upon the most advanced lesion, included tubular adenomas without high-grade dysplasia (TA), tubular adenomas with high-grade dysplasia, tubulovillous or villous adenomas without high-grade dysplasia, and tubulovillous/villous adenomas with high-grade dysplasia. Polyps with distinctly separate areas of conventional adenoma and hyperplastic polyp were classified as 'mixed hyperplastic/adenomatous polyps' and were distinguished from SSA-LD. Adenocarcinoma with no evidence of adjacent SSA was designated ADENOCA.

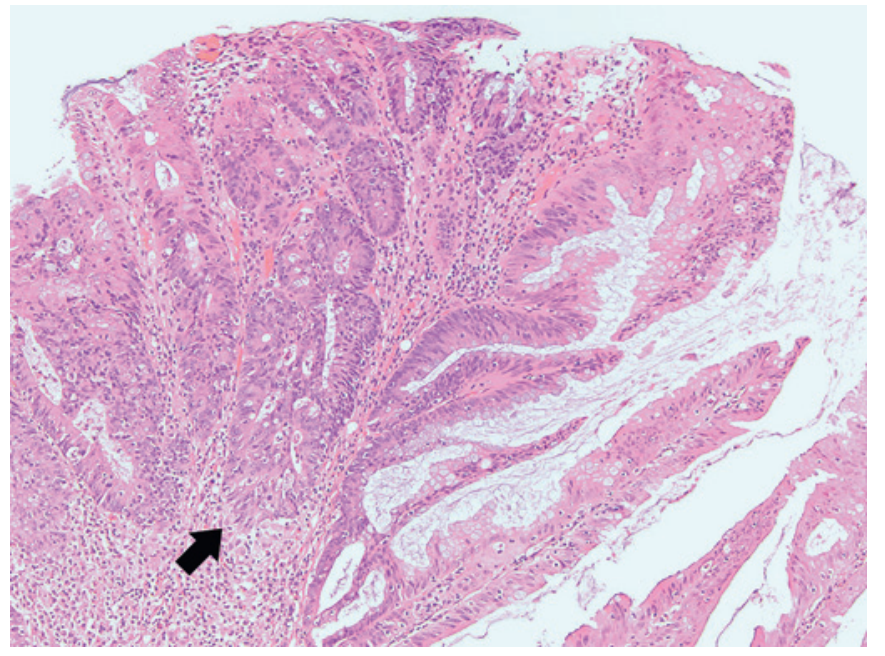

Figure 4 Sessile serrated adenoma with high-grade dysplasia. Nuclear alterations (crowding, pencillation and complex pseudostratification), apoptosis, increased mitotic activity, and architectural complexity with cribriforming (arrow) are hallmarks of high-grade dysplasia. The right portion of the photomicrograph shows a portion of the SSA with progression from a non-dysplastic epithelium to low- and high-grade dysplasia. 


\section{Data acquisition}

Diagnostic reports on 290810 colonoscopic specimens from 179111 patients generated between 1 April 2007 and 31 March 2008 were stored in a Microsoft Access Database and analysed using algorithms programmed in Visual Basic for Applications. Characterisation of data was validated through searches using structured query language. The presence or absence of each type of polyp was recorded for each specimen. When analysing patient-specific data, polyps from multiple endoscopic procedures were considered in aggregate, and the patient's age was averaged among all encounters over the 1-year study period. Statistical calculations for uncorrected $\chi^{2}$, Fisher exact, Student t and Mann-Whitney Rank Sum tests (for non-parametric data) were performed using SigmaStat v3.5 (Systat Software, Richmond, California); proportions t tests and ORs were used as appropriate.

\section{RESULTS}

The median age of all patients with colonoscopic biopsies was 59 years (average age 59.4 years). There were 88889 women (49.6\%), 90219 men (50.4\%) and three unspecified. Only 1737 patients $(1 \%)$ had biopsies from more than one endoscopy (1650 with two encounters, 77 with three encounters and 10 with four to six encounters). Mucosal polyps were present in 185469 specimens from 128106 patients. A summary of the different types of polyps found in all patients is depicted in figure 5 .

\section{SSAs}

We identified 2416 specimens from 2139 patients with at least one SSA (with or without dysplasia/carcinoma). This SSA+/group consisted of 1162 (54.3\%) women and 977 (45.7\%) men. The distribution of SSA $+/-$ in patients was: right-side $=1737$ patients $(81.2 \%)$; left-side $=240$ patients $(11.2 \%)$; both right and left-side $=70$ patients $(3.2 \%)$; not specified $=92$ patients $(4.3 \%)$. When stratifying patients with SSAs based upon the most advanced polyp (highest grade dysplasia/carcinoma) present, there were 1816 patients without dysplasia (SSA-), 257 patients with low-grade dysplasia (SSA-LD), 45 patients with high-grade dysplasia (SSA-HD) and 21 patients with adenocarcinoma arising within an SSA (SSA-CA).

Patients with SSA+/- had a median age of 62 years with a range of 23 to 95 years. The median age of patients with SSAwas 61 years, SSA-LD was 66 years, SSA-HD was 72 years, and SSA-CA was 76 years. The difference in median age between patients with SSAs without dysplasia (SSA-) and those with dysplasia or carcinoma (SSA-LD/SSA-HD/SSA-CA) was significant $(p<0.001)$; in fact, there were significant differences between the median age of most subgroups (SSA - vs SSA-LD $(p<0.001)$, SSA-LD vs SSA-HD $(p=0.002)$, SSA-HD vs SSA-CA $(p=0.07, N S))$. There were no significant differences between the median ages of men and women within each subgroup (SSA$(p=0.983)$, SSA-LD $(p=0.378)$, SSA-HD $(p=0.868, \alpha=0.050$ : $0.050)$ and SSA-CA $(p=0.526, \alpha=0.050: 0.050))$.

SSA $+/-$ were more common in women than in men (OR $=1.21,95 \%$ CI 1.11 to 1.32 ), and women comprised $53 \%$ of the SSA - group (968/1816), 57\% of the SSA-LD group (147/ $257), 69 \%$ of the SSA-HD group (31/45) and $76 \%$ of the SSA-CA group (16/21). The predominance of women in each group was significant $(p<0.001$ to $p=0.002)$, and, compared with our population undergoing colonoscopy, women were over-represented among patients with SSA-(OR 1.16, 95\% CI 1.06 to 1.27), SSA-LD (OR 1.36, 95\% CI 1.06 to 1.74 ), SSA-HD (OR $2.25,95 \%$ CI 1.20 to 4.23 ) and SSA-CA (OR 3.25, 95\% CI 1.19 to 8.87). Even among patients with SSAs, already predominantly women, women appeared to be at higher risk of developing high-grade dysplasia (OR 1.94, 95\% CI 1.0251 to 3.6708) and adenocarcinoma (OR 2.80, 95\% CI 1.02 to 7.68 ) (figure 6).

\section{Non-serrated adenomas and adenocarcinomas}

There were 117165 specimens from 86141 patients with at least one conventional adenoma with or without high-grade dysplasia/carcinoma $(\mathrm{AD}+/-)$. These patients had a median age of 62 years. The $\mathrm{AD}+/-$ group consisted of 36601 women (42.3\%) and 49540 men (57.7\%). The total number of patients, proportion of men and women, and median ages of the patients in these groups are summarised in table 1 . There were significantly fewer women in the $\mathrm{AD}+/$ - group than men $(\mathrm{OR}=0.58$, 95\% CI 0.57 to 0.59 ).

The difference in median age between patients with SSAand those with SSA-CA was 15 years. In contrast, there was only a 5 -year difference between the median age of patients with TA and those with ADENOCA (figure 7).

\section{Serrated polyps other than SSAs}

There were 77891 specimens from 64094 patients with at least one hyperplastic polyp (HP). Their median age was 59 years (9-104). HPs were less common in women (30 458; 47.5\%) than in men $(33636 ; 52.5 \%)$

TSAs with or without high-grade dysplasia were seen in 722 specimens from 709 patients. Of the 698 patients having TSAs without high-grade dysplasia, there were 355 (50.9\%) women and $343(49.1 \%)$ men with a median age of 63 years. Only 11 patients with a median age of 65 years had a TSA with highgrade dysplasia. There was no significant difference in the
Figure 5 Total number of patients with each type of polyp. The median age and percentage of women are indicated in each major box. ADENOCA, adenocarcinoma without evidence of precursor sessile serrated adenoma; HP, hyperplastic polyp; MHAP, mixed hyperplastic-adenomatous polyp; MHAPHD, mixed hyperplasticadenomatous polyp with high-grade dysplasia; SSA-, sessile serrated adenoma without dysplasia; SSA-CA, adenocarcinoma arising in a sessile serrated adenoma; SSA-HD, sessile serrated adenoma with high-grade dysplasia; SSA-LD, sessile serrated adenoma with low-grade dysplasia; TA tubular adenoma; TA-HD, tubular adenoma with high-grade dysplasia; TSA, traditional serrated adenoma; TSA-HD, traditional serrated adenoma with high-grade dysplasia; TVANA, tubulovillous and villous adenomas; TVA-HD/NA-HD, tubulovillous and villous adenomas with high-grade dysplasia. 


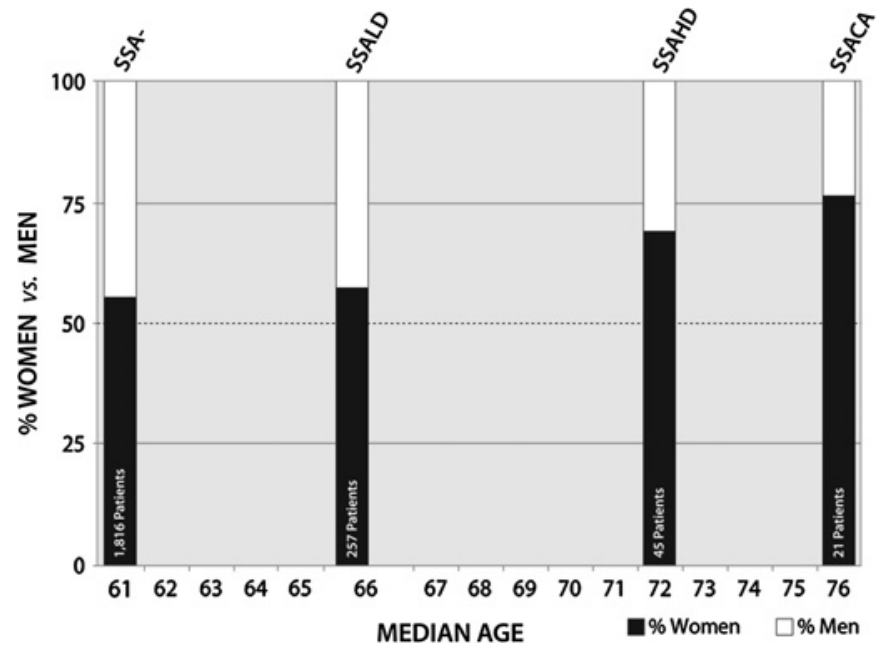

Figure 6 Demographic data for sessile serrated adenoma (SSA). SSA-, sessile serrated adenoma without dysplasia; SSACA, adenocarcinoma arising in a sessile serrated adenoma; SSA-HD, sessile serrated adenoma with high-grade dysplasia; SSA-LD, sessile serrated adenoma with low-grade dysplasia.

proportion of women versus men with TSA without (OR 1.07, $95 \%$ CI 0.92 to 1.23 ) or with high-grade dysplasia (OR $1.83,95 \%$ CI 0.48 to 6.95$)$.

MHAPs with or without high-grade dysplasia (MHAP+/-) were identified in 248 specimens from 245 patients. These polyps were found less frequently in women than men, although the difference was not significant (108 women (44.1\%) and 137 men (55.9\%); OR 0.80, 95\% CI 0.62 to 1.03; $\mathrm{p}=0.094)$ ). Patients with MHAP without high-grade dysplasia had a median age of 63.5 years while those with high-grade dysplasia had a median age of 69 years.

\section{DISCUSSION}

\section{Background, prevalence and distribution of SSAs}

This study provides unique insight regarding the epidemiology and associations of sessile serrated adenomas in a large and geographically broad-based USA patient population undergoing colonoscopy during a 12 -month period. All specimens we analysed were examined in a single laboratory where a group of pathologists dedicated to gastrointestinal pathology strives to adhere to rigorous diagnostic criteria. In order to minimise interobserver variation and increase accuracy, we use internally standardised specimen handling, diagnostic criteria and terminology. Consensus is maintained and updated through daily multiheaded microscope conferences, didactic and journal review conferences, a terminology and criteria review committee, and ongoing quality assurance activities. The joint training of pathologists and the adherence to pre-established criteria have been shown to minimise interobserver variability in the diagnosis of specific entities, despite the lack of agreement among independent pathologists, such as epithelial dysplasia in Barrett oesophagus, ${ }^{9}$ gastric atrophy ${ }^{10}$ and melanocytic dysplasia, ${ }^{11}$

Although we do not claim the complete absence of interobserver variability in this study, a major effort was made in our laboratory to achieve uniformity in the diagnosis of SSA, a relatively novel and still incompletely defined type of polyp. It is arguable that this commitment to achieve uniformity results in a set of interpretations as valid as any single observer's interpretation of the same group of polyps. As part of this effort, many SSAs without dysplasia and virtually all SSAs with dysplasia or carcinoma evaluated in the year before and during the study period were reviewed at our daily consensus conference.

Our understanding of serrated polyps has evolved since the description of serrated adenomas by Longacre and Fenoglio-Preiser ${ }^{12}$ in 1990 and the recognition of sessile serrated adenomas by Torlakovic and Snover ${ }^{8}$ in 1996. Since then, it has become clear that the predominantly left-sided 'traditional' serrated adenomas (similar to typical adenomas but with a serrated architecture and a unique cytological configuration) are relatively rare compared with the previously underappreciated sessile serrated adenomas, that resemble large hyperplastic polyps. In our study, SSAs were identified in $1.7 \%$ patients (similar to the $1.9 \%$ prevalence reported by Higuchi et al). ${ }^{12}$ SSAs comprised $3.4 \%$ of all serrated polyps, less than the $8.3 \%$ reported by Higuchi (and up to $22.6 \%$ reported in other studies). ${ }^{43-15}$ The lower proportions in this study likely reflect our conservative criteria for inclusion as SSA, since we did not include 3322 patients with 3567 serrated polyps that failed to meet all

Table 1 Demographic information of patients with selected colorectal polyps

\begin{tabular}{|c|c|c|c|c|c|}
\hline Group & Median age (range) & Women (\%) & Men (\%) & No of patients & No of specimens \\
\hline SSA- & 61 (23 to 90$)$ & $968(55.3)$ & $848(44.7)$ & 1816 & 2077 \\
\hline SSA-LD & 66 (31 to 95$)$ & $147(57.2)$ & $110(42.8)$ & 257 & 271 \\
\hline SSA-HD & 72 (50 to 87$)$ & $31(68.9)$ & $14(31.1)$ & 45 & 47 \\
\hline SSA-CA & 76 (61 to 88$)$ & $16(76.2)$ & $5(23.8)$ & 21 & 21 \\
\hline HP & 59 (9 to 104$)$ & $30458(47.5)$ & $33636(52.5)$ & 64094 & 77891 \\
\hline TSA & 63 (23 to 92$)$ & $355(50.9)$ & $343(49.1)$ & 698 & 711 \\
\hline TSAHD & 65 (53 to 78 ) & $8(72.7)$ & $3(27.3)$ & 11 & 11 \\
\hline TA & 62 (6 to 106) & $31841(42.2)$ & $43648(57.8)$ & 75489 & 105308 \\
\hline TVA/VA & 64 (17 to 99$)$ & $2991(45.2)$ & $3625(54.8)$ & 6616 & 7554 \\
\hline TAHD & 66 (37 to 92) & $351(41.0)$ & $506(59.0)$ & 857 & 969 \\
\hline TVAHD/VAHD & 65 (25 to 94$)$ & $478(45.2)$ & $580(54.8)$ & 1058 & 1144 \\
\hline ADENOCA & 67 (24 to 99$)$ & $940(44.3)$ & $1181(55.7)$ & 2121 & 2190 \\
\hline MHAP & 63.5 (32 to 88 ) & $106(44.5)$ & $132(55.5)$ & 238 & 241 \\
\hline MHAPHD & $69(51$ to 80$)$ & $1(16.7)$ & $5(88.3)$ & 6 & 7 \\
\hline
\end{tabular}

ADENOCA, adenocarcinoma without evidence of precursor sessile serrated adenoma; HP, hyperplastic polyp; MHAP, mixed hyperplastic-adenomatous polyp; MHAPHD, mixed hyperplasticadenomatous polyp with high-grade dysplasia; SSA-, sessile serrated adenoma without dysplasia; SSA-CA, adenocarcinoma arising in a sessile serrated adenoma; SSA-HD, sessile serrated adenoma with high-grade dysplasia; SSA-LD, sessile serrated adenoma with low-grade dysplasia; TA, tubular adenoma; TA-HD, tubular adenoma with high-grade dysplasia; TSA, traditional serrated adenoma; TSA-HD, traditional serrated adenoma with high-grade dysplasia; TVA/VA, tubulovillous and villous adenomas; TVA-HD/NA-HD, tubulovillous and villous adenomas with highgrade dysplasia. 


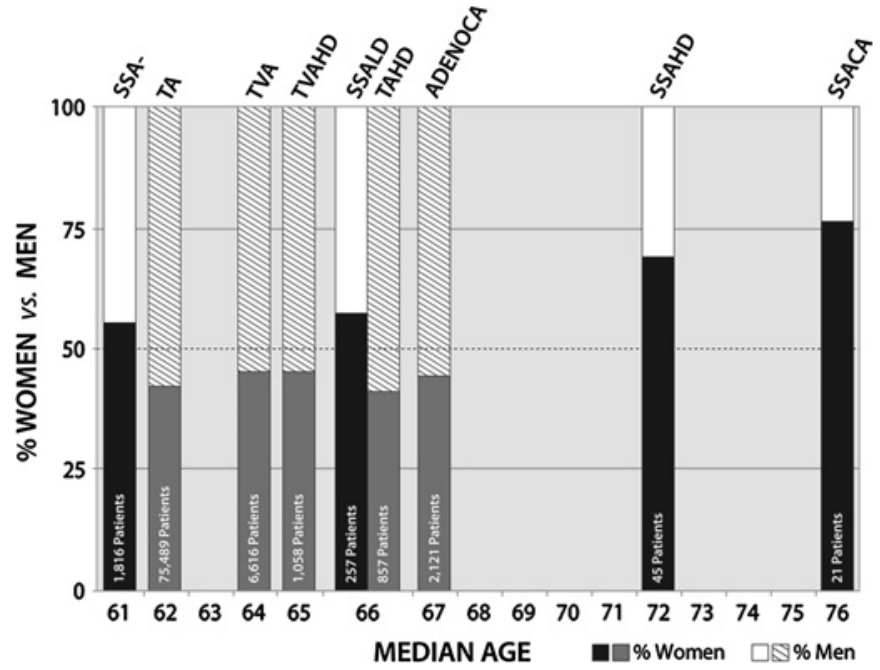

Figure 7 Comparison of demographic data for sessile serrated adenoma (SSA) with conventional adenomatous lesions. ADENOCA, adenocarcinoma without evidence of precursor sessile serrated adenoma; MHAPHD, mixed hyperplastic-adenomatous polyp with highgrade dysplasia; SSA-, sessile serrated adenoma without dysplasia; SSACA, adenocarcinoma arising in a sessile serrated adenoma; SSAHD, sessile serrated adenoma with high-grade dysplasia; SSALD, sessile serrated adenoma with low-grade dysplasia; TA, tubular adenoma; TAHD, tubular adenoma with high-grade dysplasia; TVA, tubulovillous adenomas; TVAHD, tubulovillous adenomas with high-grade dysplasia.

histopathological criteria we set forth for the unequivocal diagnosis of SSA. Had we included them in the SSA group, our rates would be closer to Higuchi's at $3.2 \%$ and $8.4 \%$, respectively, but the age and gender differences between those with and without dysplasia would not be significantly affected. Our findings also support the previously reported right-sided and female predominance of SSAs. ${ }^{3} 81316$

\section{Dysplasia and carcinoma arising in SSAs}

We identified low-grade dysplasia in $12.0 \%$ of our patients with SSAs, high-grade dysplasia in $2.1 \%$ and adenocarcinoma in $1.0 \%$. Remarkably similar proportions of our patients with conventional adenomatous polyps had high-grade dysplasia (2.2\%) and adenocarcinoma $(2.5 \%)$. The percentage of women increased progressively from $53 \%$ for SSAs without dysplasia to $57 \%$ for SSA with low-grade dysplasia, 69\% for SSA with high-grade dysplasia and 76\% for SSA with adenocarcinoma. In contrast, no significant gender differences were present in the progression of adenomas to high-grade dysplasia and carcinoma (figure 2).

Over the last several years, it has been suggested that SSAs may progress to adenocarcinoma more rapidly than conventional adenomas. $^{2} 35$ These concerns have led to the suggestion that screening guidelines based upon the progression rates of conventional adenomas may not be adequate for SSAs, ${ }^{17}$ and surgery for cancer prevention has been proposed for patients whose serrated lesions cannot be completely excised endoscopically. ${ }^{16}$

Our study showed a difference of 5 years between the median age of patients with SSA and the median age of patients with SSA with low-grade dysplasia; 11 years between SSA and SSA with high-grade dysplasia; and 15 years between SSA and SSA with carcinoma. Clearly, no study that lacks extended follow-up of patients can accurately determine the rate of progression of a condition; nevertheless, the considerable age differences we detected could be interpreted to indicate that SSAs progress in a stepwise fashion through intermediate stages of low- and highgrade dysplasia. By comparison, the difference in median age

\section{Take-home messages}

Sessile serrated adenomas/polyps occur in $1.7 \%$ of patients with colonic epithelial polyps, are more common in women and are detected more frequently in the right colon.

- These serrated polyps appear to undergo a stepwise progression to dysplasia and carcinoma over a time period that is two to three times longer than that in conventional adenomas.

- The progression to dysplasia and carcinoma occurs disproportionately among women.

between our patients with tubular adenomas (without high-grade dysplasia) and our patients with adenocarcinoma unrelated to SSA was only 5 years. Similar evidence suggesting a prolonged rather than shortened time frame for the development of adenocarcinoma arising within SSAs comes from a study of 91 patients with MSI-H adenocarcinomas, in which the mean and median intervals between removal of a 'hyperplastic-like' polyp and subsequent mismatch repair-deficient adenocarcinoma were 7.3 and 6.5 years, respectively. ${ }^{4}$ In a subsequent study of six SSA with carcinoma by the same author, the mean patient age was 69.5 years (range 57.1-83.9 years). ${ }^{2}$ It is arguable that the evidence presented in this and other studies, suggesting a more indolent course for these lesions, especially without dysplasia, supports the notion that they are best referred to as 'sessile serrated polyps.' It is also conceivable, however, that while the majority of SSAs follow a stepwise progression to adenocarcinoma over many years, a subset of SSAs may gain specific molecular alterations that cause a more rapid progression to adenocarcinoma. Indeed, Oono et al ${ }^{16}$ recently reported endoscopic progression of a SSA to early invasive carcinoma within a period of 8 months. For this reason, some authors will likely prefer the term 'Sessile serrated adenoma.'

While it would be premature to suggest that no aggressive treatment is needed for well-sampled, non-dysplastic and lowgrade dysplastic SSAs based on these findings, we would hope that these data provide a stimulus for the development of controlled follow-up studies.

Acknowledgements The authors wish to thank S Gupta, Division of Gastroenterology, UTSW Medical Center at Dallas, for helpful insights and suggestions.

\section{Competing interests None.}

Ethics approval Ethics approval was provided by the Caris Diagnostics Institutional Review Board.

Contributors RHL designed the study, interpreted the data, cowrote the manuscript and prepared one figure. RMG designed the study, analysed the data, interpreted the data, cowrote the manuscript and prepared one figure. CMS designed the study, the prepared database for analysis, analysed the data, participated in data interpretation cowrote the manuscript and prepared two figures.

Provenance and peer review Not commissioned; externally peer reviewed.

\section{REFERENCES}

1. Snover DC, Jass JR, Fenoglio-Preiser C, et al. Serrated polyps of the large intestine: a morphologic and molecular review of an evolving concept. Am J Clin Pathol 2005; 124:380-91.

2. Goldstein NS. Small colonic microsatellite unstable adenocarcinomas and highgrade epithelial dysplasias in sessile serrated adenoma polypectomy specimens: a study of eight cases. Am J Clin Pathol 2006;125:132-45.

3. Sheridan TB, Fenton H, Lewin MR, et al. Sessile serrated adenomas with low- and high-grade dysplasia and early carcinomas: an immunohistochemical study of serrated lesions 'caught in the act'. Am J Clin Pathol 2006;126:564-71. 
4. Goldstein NS, Bhanot P, Odish E, et al. Hyperplastic-like colon polyps that preceded microsatellite-unstable adenocarcinomas. Am J Clin Pathol 2003;119:778-96.

5. Jass JR. Serrated route to colorectal cancer: back street or super highway? J Pathol 2001:193:283-5.

6. Goldstein NS. Clinical significance of (sessile) serrated adenomas: Another piece of the puzzle. Am J Clin Pathol 2005:123:329-30.

7. Haramis AP, Beghtel $\mathrm{H}$, van den Born $\mathrm{M}$, et al. De novo crypt formation and juvenile polyposis on BMP in mouse intestine. Science 2004;303:1684-6.

8. Torlakovic E, Snover DC. Serrated adenomatous polyposis in humans Gastroenterology 1996;110:748-55.

9. Montgomery E, Bronner MP, Goldblum JR, et al. Reproducibility of the diagnosis of dysplasia in Barrett esophagus: a reaffirmation. Hum Pathol 2001;32:368-78.

10. Rugge M, Correa P, Dixon MF, et al. Gastric mucosal atrophy: interobserver consistency using new criteria for classification and grading. Aliment Pharmacol Ther 2002:16:1249-59
11. Weinstock MA, Barnhill RL, Rhodes AR etal. Reliability of the histopathologic diagnosis of melanocytic dysplasia the dysplastic nevus panel. Arch Dermatol 1997;133:953-8.

12. Longacre TA, Fenoglio-Preiser CM. Mixed hyperplastic adenomatous polyps/ serrated adenomas. A distinct form of colorectal neoplasia. Am J Surg Pathol 1990; 14:524-37

13. Higuchi T, Sugihara K, Jass JR. Demographic and pathological characteristics of serrated polyps of colorectum. Histopathology 2005;47:32-40.

14. Torlakovic E, Skovlund E, Snover DC, et al. Morphologic reappraisal of serrated colorectal polyps. Am J Surg Pathol 2003;27:65-81.

15. Montgomery E. Serrated colorectal polyps: emerging evidence suggests the need for a reappraisal. Adv Anat Pathol 2004;11:143-9.

16. Oono Y, Fu K, Nakamura $\mathrm{H}$, et al. Progression of a sessile serrated adenoma to an early invasive cancer within 8 months. Dig Dis Sci 2009;54:906-9.

17. Freeman HJ. Heterogeneity of colorectal adenomas, the serrated adenoma, and implications for screening and surveillance. World J Gastroenterol 2008:14:3461-3. 